\title{
STABILITY ANALYSIS AND DESIGN OF ALGORITHMIC TUNED PID CONTROLLER FOR BIO-REACTOR
}

\author{
Abhishek $\mathbf{V}^{1}$, Arul Ebenezer $\mathbf{E}^{2}$ \\ ${ }^{1} U G$ student, Department of Electronics and Instrumentation Engineering, St. Joseph's College of Engineering, \\ Chennai 600 119, India \\ ${ }^{2} U G$ student, Department of Electronics and Instrumentation Engineering, St. Joseph's College of Engineering, \\ Chennai 600 119, India
}

\begin{abstract}
The paper proposes a detailed analysis of various intelligent algorithms for the application of tuning PID controllers for time delayed model with a specific stability analysis on the unstable phase of a Bio- reactor system. The tuning process is focused mainly to search the optimal controller parameters $\left(K_{p}, K_{i}, K_{d}\right)$ by minimizing the performance criterion and with the help of the objective function. Relative studies on various cost functions like integral of squared error (ISE), integral of absolute error (IAE), integrated time squared error (ITSE), integrated time absolute error (ITAE) have also been performed for the tuned system. The stability analysis for the algorithmic tuned PID controller by Nyquist plot is also performed for the various working phases of the considered bio reactor model. The simulation results show that the proposed tuning approach provides superior results such as smooth set point tracking, error minimization and increased stable response.
\end{abstract}

Keywords: stability, PID, PSO, BFO, FF, bio reactor

\section{INTRODUCTION}

Despite significant developments in advanced process control schemes such as Model Predictive Control (MPC), Internal Model Control (IMC), and Sliding Mode Control (SMC), PID controllers are still widely used in industrial control system where reference tracking and disturbance rejection are a major task

Proportional + Integral + Derivative (PID) controllers are widely used in industrial applications to provide optimal and robust performance for stable, unstable and non-linear processes. It can be easily implementable in analog or digital form. Further, it supports tuning and online retuning based on the performance requirement of the process to be controlled. The literature gives details about various theoretical studies on fine tuning of PID controllers for open-loop unstable system [4, 10, and 12]. Recently Rajinikanth and Latha have proposed the optimization of the PID controller parameters using soft computing method [14]. Recently, Ebenezer and Abhishek have proposed PSO based tuning method for a class of stable and unstable systems [2].

Passino devised the Bacterial Foraging Algorithm in the year 2002 [11]. Ali and Majhi have proposed PID controller tuning for a class of time delayed stable systems using Bacterial Foraging Optimization (BFO) algorithm [1]. Korani et al. presented a comparative analysis on PSO, BFO and hybrid algorithm based PID controller for a stable system [16]. It has been modified and improved by Christian and Maria [5]. The fire fly algorithm as devised by Yang [18] provides an adaptive approach towards the optimization view with a varied nature differing from the usual approaches. Several researches and modifications have been proposed on this algorithm recently [6 and 17]. It has also been applied to several design constraints and has been successfully implemented [3 and 7].

The above algorithms have been implemented in several applications. A benchmark problem of Bio-reactor control has been taken in several situation to perform an analysis of the algorithms over the previous years. Identification and modelling of the Bio-reactor has been proposed by Pramod and Chidambaram [15]. Genetic Algorithm based tuning approach has been applied to this problem by Kumar and Jain in 2008 [9]. Rajinikanth and Latha have proposed a method to tune the bio-reactor in the year 2010 [13].

In this work, the PID controller parameter tuning is proposed for bio-reactor model using Particle Swarm Optimization (PSO) algorithm introduced by Kennedy and Eberhart [8], Bacterial Foraging Algorithm by Passino [11], Firefly Algorithm by Yang [18]. A brief comparison is done on the three methods. The above algorithms are biologically inspired algorithms and are successfully used for engineering applications due to their high computational efficiency, easy implementation and stable convergence. In this work, to evaluate the performance of the proposed methods, a simulation study is carried out using a bioreactor system model and to analyze the stability of the tuned model, stability analysis is done using Nyquist plot.

In this work, the PID controller parameter tuning is performed for the stable and unstable phases of the bio reactor system using three different algorithms individually and Our objective is to reduce the errors ISE- Integral 
Squared Error, IAE- Integral Absolute Error, ITAE- Integral Time Absolute Error, ITSE- Integral Time Squared Error so that the response of the system would settle at the required set-point. These errors are referred to as Performance Criteria as they finally decide how efficient the controller is in reducing the errors. Another advantage of the algorithmic usage in the tuning consideration is that, a detailed mathematical description of the system is not required and the parameters can be found in an easy manner.

\section{METHODOLOGY}

\subsection{Algorithms}

\subsubsection{Particle Swarm Optimization}

PSO- Particle Swarm Optimization. This meta-heuristic algorithm was originally proposed and developed by Kennedy, Eberhart and Shi. It makes very few or no assumptions about the given problem. It can also search very large spaces. However, like any meta-heuristic algorithm, PSO does not guarantee any optimal solution is found. Therefore, we search for the solution among the candidate solutions for the given problem

The candidate solutions in the search space are referred to as particles. These are made to move around the search space based upon their local best position and global best positions which is iteratively updated in case of improvements.

Let us consider the function ' $\mathrm{f}: \mathrm{R}^{\mathrm{n}} \rightarrow \mathrm{R}$ ' as the cost function to be minimized. The particle (candidate solution) as a vector of real numbers is the argument to this function. As PSO is not slope based, gradient of ' $\mathrm{f}$ ' is not known. Our goal is to find a solution ' $\mathrm{a}$ ' such that $\mathrm{f}(\mathrm{a}) \leq \mathrm{f}(\mathrm{b}) \forall \mathrm{b}$ in the search space.

If $S$ is number of particles in swarm, each with position $x_{i}$ $\in \mathrm{R}^{\mathrm{n}}$ and velocity $\mathrm{v}_{\mathrm{i}} \in \mathrm{R}^{\mathrm{n}}$. Let $\mathrm{p}_{\mathrm{I}}$ be local best and $\mathrm{g}$ be global best, then

- $\quad$ For all S particles starting from 1, initialize position with an uniformly distributed vector: $\mathrm{x}_{\mathrm{i}} \sim \mathrm{U}\left(\mathrm{b}_{\mathrm{lo}}, \mathrm{b}_{\mathrm{up}}\right)$, where $b_{\text {lo }}, b_{\text {up }}$ are lower and upper search space boundaries.

- Initialize $\mathrm{p}_{\mathrm{i}}$ to $\mathrm{x}_{\mathrm{i}}$

- If $\left(\mathrm{f}\left(\mathrm{p}_{\mathrm{i}}\right)<\mathrm{f}(\mathrm{g})\right)$, update the swarm's best known position: $\mathrm{g} \leftarrow \mathrm{p}_{\mathrm{i}}$

- Initialize the particle's velocity: $\mathrm{v}_{\mathrm{i}} \sim \mathrm{U}\left(-\left|\mathrm{b}_{\mathrm{up}}-\mathrm{b}_{\mathrm{lo}}\right|\right.$, $\left.\left|b_{\text {up }}-b_{\text {lo }}\right|\right)$ Until the numbers of iterations specified are completed or an adequate solution is found, repeat

- For all $\mathrm{S}$ particles starting from 1, pick random numbers: $r_{p}, r_{g} \sim U(0,1)$

- For each dimension $\mathrm{d}=1$ to $\mathrm{n}$, update

$$
\mathbf{v}_{\mathrm{i}, \mathrm{d}} \leftarrow \omega \mathbf{v}_{\mathrm{i}, \mathrm{d}}+\varphi_{\mathrm{p}} r_{\mathrm{p}}\left(\mathbf{p}_{\mathrm{i}, \mathrm{d}}-\mathbf{x}_{\mathrm{i}, \mathrm{d}}\right)+\varphi_{\mathrm{g}} r_{\mathrm{g}}\left(\mathbf{g}_{\mathrm{d}}-\mathbf{x}_{\mathrm{i}, \mathrm{d}}\right)
$$

- Update the particle's position: $\mathbf{x}_{\mathrm{i}} \leftarrow \mathbf{x}_{\mathrm{i}}+\mathbf{v}_{\mathrm{i}}$

- If $\left(f\left(\mathbf{x}_{\mathrm{i}}\right)<f\left(\mathbf{p}_{\mathrm{i}}\right)\right)$ do:

○ Update the particle's best known position: $\mathbf{p}_{\mathrm{i}}$ $\leftarrow \mathbf{x}_{\mathrm{i}}$
- If $\left(f\left(\mathbf{p}_{\mathrm{i}}\right)<f(\mathbf{g})\right)$ update the swarm's best known position: $\mathbf{g} \leftarrow \mathbf{p}_{\mathrm{i}}$

- Now $\mathbf{g}$ holds the best found solution.

The parameters $\omega, \varphi_{\mathrm{p}}$, and $\varphi_{\mathrm{g}}$ are selected by the practitioner and control the behavior and efficacy of the PSO method The following algorithms are only explained in an outlined way.

\subsubsection{Bacterial Foraging Optimization}

The bacterial foraging optimization (BFO) proposed by Passino in the year 2002 [11] is based on natural selection that tends to eliminate animals with poor foraging strategies. Over certain real-world optimization problems, BFO has been reported to outperform many powerful optimization algorithms in terms of convergence speed and final accuracy

The BFO algorithm which mimics the above four mechanisms is present Chemo taxis: Represents the step size of the bacteria.

Swarming: Grouping of the bacteria which allows them to move in a concentric pattern. Reproduction: Maintains the population in the swarm

Elimination and Dispersal: The BFO algorithm makes some bacteria to get eliminated and dispersed with probability to ensure that the bacteria do not get trapped into a local optimum instead of the global optima.

\subsubsection{Fire Fly Algorithm}

We can idealize some of the flashing characteristics of fireflies so as to develop firefly-inspired algorithms. Flashing characteristics of fireflies is used to develop fireflyinspired algorithm. Firefly Algorithm (FA or FFA) developed by Xin-She Yang at Cambridge University in 2007, use the following three idealized rules:

- All the fireflies are unisex so it means that one firefly is attracted to other fireflies irrespective of their sex.

-Attractiveness and brightness are proportional to each other, so for any two flashing fireflies, the less bright one will move towards the one which is brighter. Attractiveness and brightness both decrease as their distance increases. If there is no one brighter than other firefly, it will move randomly.

- The brightness of a firefly is determined by the view of the objective function. For a maximization problem, the brightness is simply proportional to the value of the objective function. Other forms of the brightness could be defined in an identical way to the fitness function in genetic algorithms.

All the above considered algorithms are converted into suitable pseudo code and are linked with model using the MATLAB to perform the simulated behavior. 


\section{SYSTEM MODEL DESCRIPTION}

The below represented bioreactor model is widely considered by the researchers for performing the simulation based study $[15,20]$. Bioreactor cultivation operation and nutrient feeding has significant impact on the outcome of the bioreactor. The reactor is a closed system and is highly dynamic in nature. Medium composition changes continuously with time to produce biomass and metabolites. The major problem of batch cultivation is high non production time and low productivity. In order to increase product formation and productivity substrate concentration in the batch could be taken higher, however sometimes it leads to substrate inhibition.

This problem of high substrate concentration can be eliminated by gradual feeding of the substrate. The biomass concentration varies accordingly with reaction rate of the biosynthesis. During the reaction phase, a controlled environment is maintained in the bioreactor maintaining a constant $\mathrm{pH}$, temperature, agitation rate and dissolved oxygen tension to attain better growth of microbes.

In order to illustrate the system representation, a mathematical model- based simulation of actual bioreactor runs suggest how process variables such as substrate and product concentrations change and how nutrient feeding should be "tuned" with respect to time, pattern, concentration, and composition to elicit a desired response. The modelling of the cellular productivity in the bioreactor produces a formidable challenge because of many inherent high degree nonlinearities.

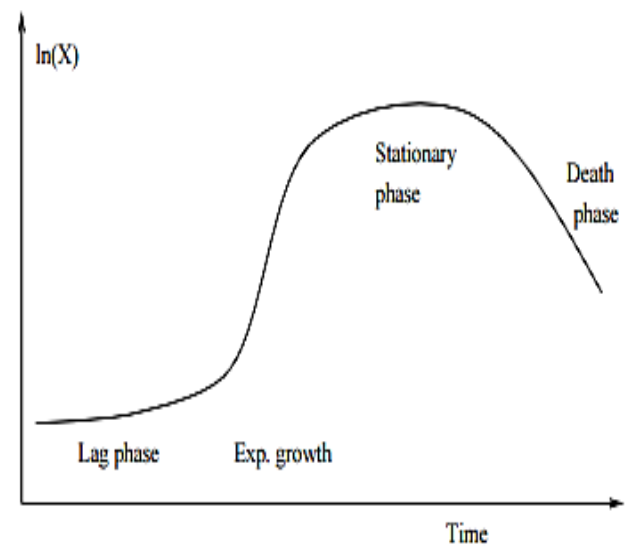

Fig 1: Phases in a bio reactor

Lag phase and Death phase do not require explicit control requirements. Exponential growth phase and stationary phase require proper control so as to produce optimal productivity. It is notable that the stationary phase is a stable one and the exponential growth phase is an unstable one. Considering the exponential growth phase which can be expressed as

$$
\frac{d X(t)}{d x}=\mu X(t)
$$

Where $\mu$ is denoted as the specific growth rate, "rate of increase in cell concentrations per unit cell concentrations"

Often the specific growth rate $\mu$ depends on the substrate concentration $\mathrm{S}$. The following equation specifies the relation between the growth phase and the substrate concentration $\mathrm{S}$, which is given by the commonly named Monod function represented as

$$
\mu(S)=\mu_{\max } \frac{S}{K_{S}+S}
$$

$\mu_{\max }$ is the maximum specific growth rate

$\mathrm{S}$ is the concentration of growth limiting substrate

$\mathrm{K}_{\mathrm{s}}$ is the half saturation

Thus by devising the mathematical modelling of the bio reactor as developed by $[15,20]$, we get the transfer function model for two of the operating regions as described below

\subsubsection{Operating Ranges of the Bio Reactor:}

\section{Regional Estimation}

$\begin{array}{ll}\begin{array}{l}\text { OPERAT } \\ \text { ING }\end{array} & \text { TRANSFER FUNCTION } \\ \text { REGION } & \\ \begin{array}{l}\text { Stationary } \\ \text { phase }\end{array} & G(s) \\ & =\frac{(-1.53 s-0.4588) e^{-0.1 s}}{s^{2}+2.564 s+0.6792} \\ \begin{array}{l}\text { Growth } \\ \text { phase }\end{array} & \begin{array}{l}G(s) \\ \end{array} \\ & =\frac{(-0.9951 s-0.2985) e^{-0.1 s}}{s^{2}+0.1302 s-0.0509}\end{array}$

Now considering the above two operating ranges, the growth phase produces a challenging unstable quality and the stationary phase produces a settling stable solution. The dilution rate is taken as the manipulated variable to control the cell mass concentration at the unstable steady state. Thus the below considered simulations are done for the above bioreactor model having operations in both the phases.

\section{RESULTS AND DISCUSSIONS}

\subsection{Algorithm Analysis and Design of PID for Stationary Phase}

The stationary phase of the bioreactor which has an operation in the stable region, the simulations of the model which is devised in the MATLAB Simulink are linked with the algorithm and a continuous iterations are loped.

The following simulation results are obtained for the Fire Fly algorithm for the stationary phase.

For the stationary phase, the transfer function of the bioreactor as described above, a MATLAB based simulation model is developed and all the above mentioned algorithms were implemented to solve the optimization problem of which the Fire Fly (FF) algorithm proved to be better when 
compared with the other. Due to space constraints we have depicted the results of FF which is used to obtain optimal values of $\mathrm{K}_{\mathrm{p}}, \mathrm{K}_{\mathrm{i}}$ and $\mathrm{K}_{\mathrm{d}}$.

Table 1: Firefly Algorithm based PID controller tuning and performance parameters- Stationary phase

\begin{tabular}{|c|c|c|c|c|c|c|c|}
\hline $\begin{array}{c}\text { Iter } \\
\text { response } \\
\text { no. }\end{array}$ & $\mathbf{K}_{\mathbf{p}}$ & $\mathbf{K}_{\mathbf{i}}$ & $\mathbf{K}_{\mathbf{d}}$ & 0vershoot & $\mathrm{T}_{\mathrm{r}}$ & $\mathrm{T}_{\mathbf{s}}$ & $\mathrm{T}_{\mathbf{p}}$ \\
\hline 1 & 0.5205 & -0.2059 & -0.0857 & 0.00 & 7.85 & 29.30 & $\mathrm{NA}$ \\
\hline 2 & -0.2926 & -0.8383 & -0.0037 & 0.00 & 3.47 & 11.90 & $\mathrm{NA}$ \\
\hline 3 & -0.0798 & -0.3018 & -0.1842 & 0.00 & 9.97 & 31.10 & $\mathrm{NA}$ \\
\hline 4 & 0.6841 & -0.4886 & -0.2512 & 0.16 & 1.90 & 19.90 & 6.20 \\
\hline $\mathbf{5}$ & $\mathbf{- 0 . 0 1 1 3}$ & $-\mathbf{- 0 . 8 5 0 3}$ & $-\mathbf{0 . 0 6 0 3}$ & $\mathbf{0 . 0 0}$ & $\mathbf{2 . 7 0}$ & $\mathbf{8 . 7 0}$ & $\mathbf{N A}$ \\
\hline
\end{tabular}

Several iterations were run and 5 best values are tabulated below. Performance criteria such as ISE, IAE, ITSE and ITAE are also evaluated

Based on the values obtained, the best values were chosen based on the performance evaluation criteria and controller output effectiveness. The $5^{\text {th }}$ value in the tabular column (in Bold) is chosen as the optimal solution for the given system, since it outperforms other values in all criteria.

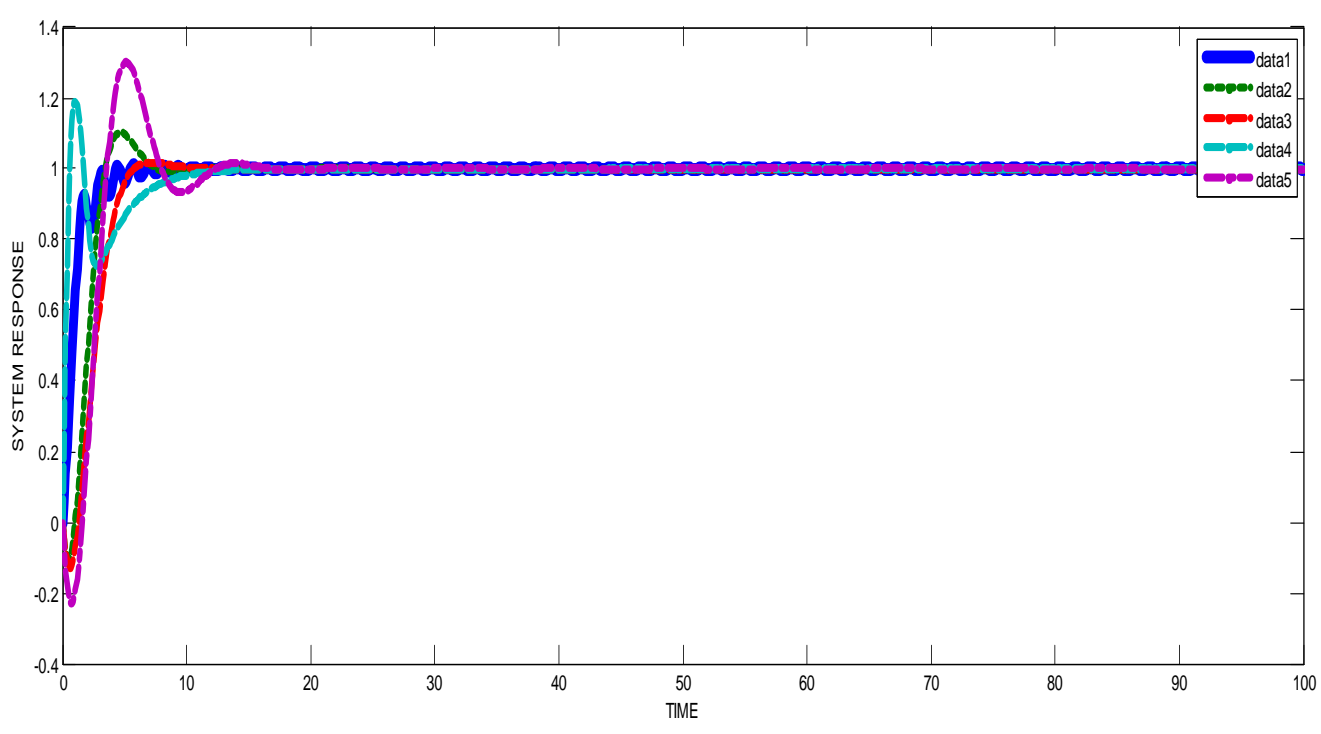

Fig 2: Response of stationary phase for Firefly Algorithm tuned PID controller

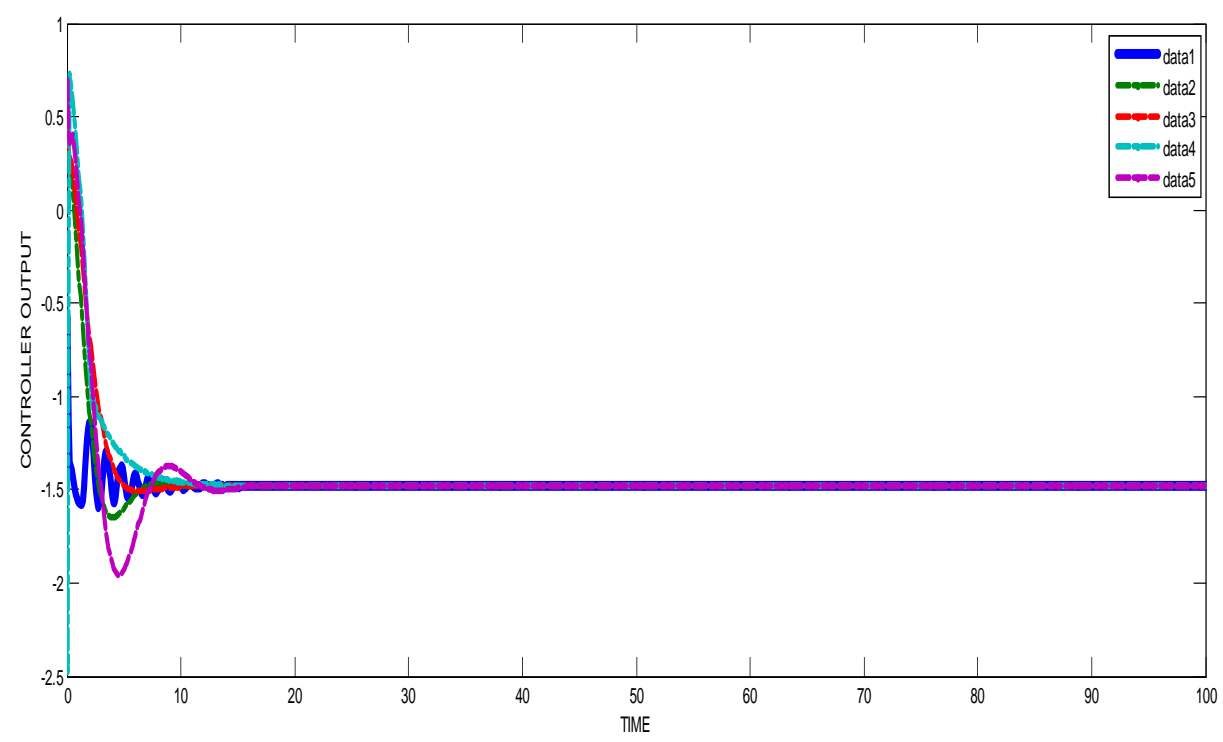

Fig 3: Controller output for Firefly Algorithm tuned PID controller- Stationary phase 
The figure 2 and 3 represents the system response and the controller output of the fire fly tuned system respectively.

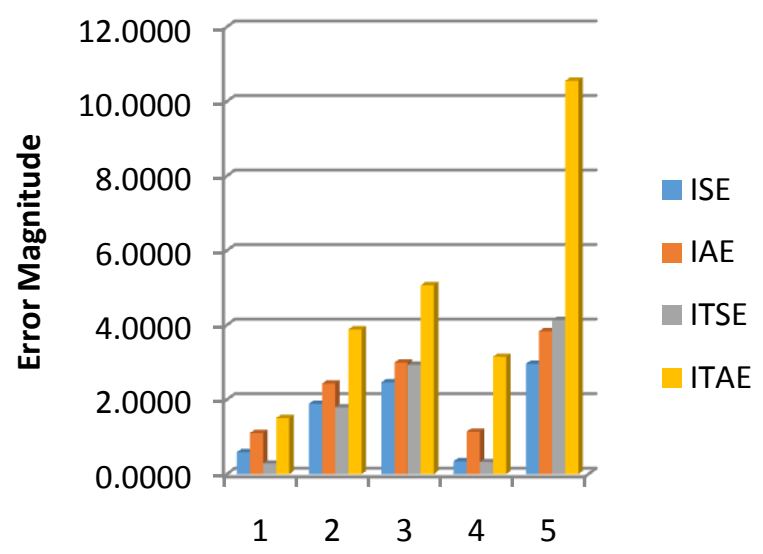

Fig 4: Performance Criteria for Firefly based PID controllerStationary phase

The above represents the fire fly algorithm involvement in the tuning of PID controller in the stationary phase of the bio reactor. From the figure 4 , the performance criterions, which mainly decide the controller efficiency is found and our main focus is to reduce them such that the performance is increased when going in for the time delayed system.

Based on the simulated results, a simple comparison is made to determine the best algorithm among the three algorithms considered: PSO, BFO and Firefly Algorithms for the Stationary phase of the bio-reactor

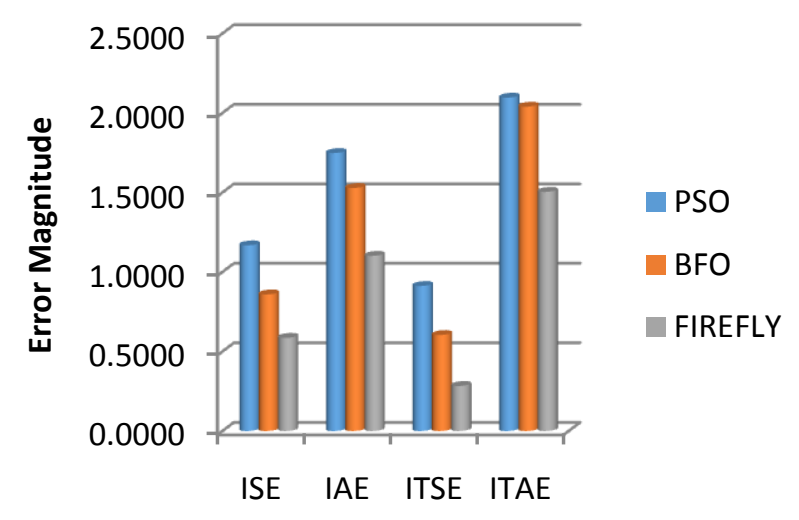

Fig 5: overall Performance comparison- Stationary phase

Based on the above depicted results the following conclusions for the stationary phase can be depicted as the Fire Fly (FF) algorithm provides the least error value when compared with the other algorithms (i.e.) it exhibits good performance criteria when Bacterial Foraging Optimization(BFO) and Particle Swarm Optimization (PSO). On the basis of the performance criterion a solid establishment has been laid that the FF algorithm works well under the stationary phase or the stable system consideration with the delay involvement.

\subsection{Controller Design for Growth Phase}

The growth phase of the bioreactor is one of the challenging effort to achieve a stability in the operation due to the unstable effects produced causing a difficulty in terms of controlling. The following simulations were done and considered for the growth phase and the stability is analyzed with the help of Nyquist plot.

\subsubsection{PSO Algorithm Based Tuning}

The stationary phase of the bioreactor which has an operation in the stable region, the simulations of the model which is devised in the MATLAB Simulink are linked with the algorithm and a continuous iterations are looped.

The following simulation results are obtained for the PSO algorithm for the growth phase of the second order time delayed system.

For the growth phase, the transfer function of the bio-reactor as described above, a MATLAB based simulation model is developed and all the above mentioned algorithms were implemented to solve the optimization problem of which the PSO algorithm proved to be better when compared with the other. Due to space constraints we have depicted the results of PSO only which is used to obtain optimal values of $\mathrm{K}_{\mathrm{p}}, \mathrm{K}_{\mathrm{i}}$ and $K_{d}$. when compared with the other considered algorithms.

For the growth phase transfer function of the bio-reactor as described in the previous chapter, a MATLAB based simulation model is developed and the PSO algorithm is used to obtain optimal values of $\mathrm{K}_{\mathrm{p}}, \mathrm{K}_{\mathrm{i}}$ and $\mathrm{K}_{\mathrm{d}}$.

Several iterations were run and 6 best values are tabulated below. Performance criteria such as ISE, IAE, ITSE and ITAE are also evaluated.

Table 2: PSO Algorithm based PID controller tuning and performance parameters- Growth phase

\begin{tabular}{|c|c|c|c|c|c|c|c|c|}
\hline $\begin{array}{c}\text { Iter } \\
\text { response } \\
\text { no. }\end{array}$ & $\mathbf{K}_{\mathbf{p}}$ & $\mathbf{K}_{\mathbf{i}}$ & $\mathbf{K}_{\mathbf{d}}$ & 0vershoot & $\mathbf{T}_{\mathbf{r}}$ & $\mathbf{T}_{\mathbf{s}}$ & $\mathbf{T}_{\mathbf{p}}$ \\
\hline 1 & -0.6737 & -0.4229 & 0.1013 & 0.509 & 1.50 & 29.93 & 3.22 \\
\hline 2 & -0.9717 & -0.3537 & -0.5188 & 0.338 & 1.93 & 24.11 & 4.22 \\
\hline 3 & -0.6962 & -0.1433 & -0.4183 & 0.390 & 2.70 & 37.22 & 6.12 \\
\hline 4 & -0.6609 & -0.1582 & -0.8445 & 0.424 & 3.23 & 50.00 & 7.22 \\
\hline $\mathbf{5}$ & $\mathbf{- 0 . 9 9 7 8}$ & $\mathbf{- 0 . 2 0 4 0}$ & $\mathbf{- 0 . 1 8 9 8}$ & $\mathbf{0 . 2 8 3}$ & $\mathbf{1 . 8 3}$ & $\mathbf{2 7 . 6 1}$ & $\mathbf{4 . 2 2}$ \\
\hline 6 & -0.6483 & -0.2521 & -0.4166 & 0.473 & 2.43 & 41.23 & 5.22 \\
\hline
\end{tabular}

Based on the values obtained, the best values were chosen based on the performance evaluation criteria and controller output effectiveness. The $5^{\text {th }}$ value in the tabular column (in Bold) is chosen as the optimal solution for the given system. 


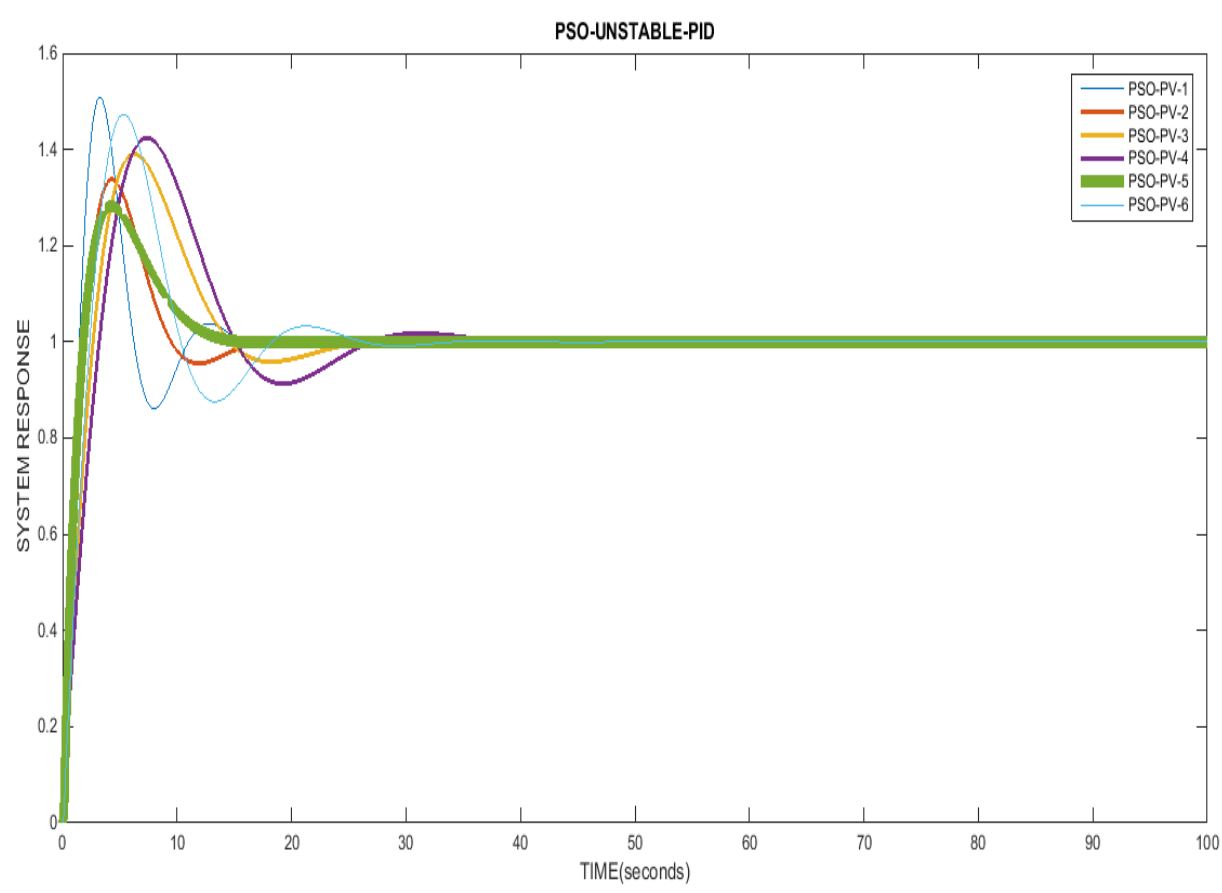

Fig 6: System Response of growth phase for PSO Algorithm tuned PID controller

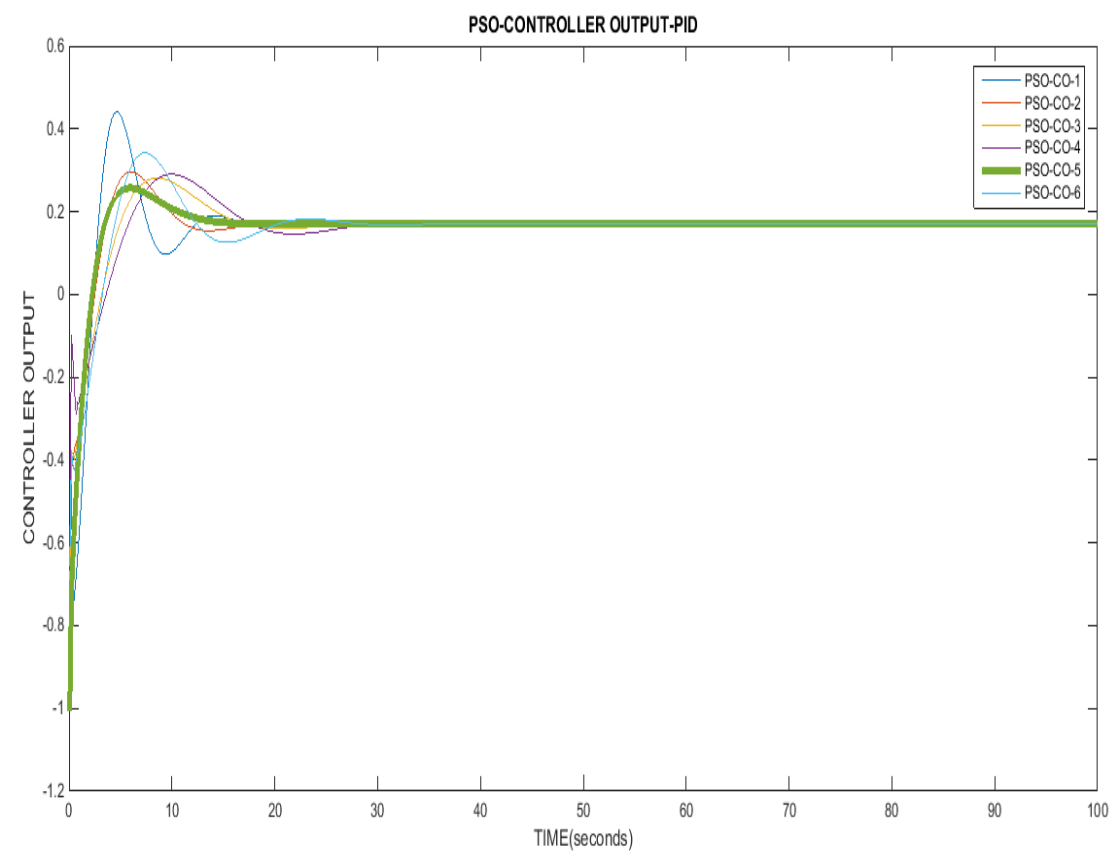

Fig 7: Controller output for PSO Algorithm tuned PID controller- Growth phase

Figure 6 and 7, represents the system's response and the controller output for the algorithmic tuned PID controller for the growth phase of the bio reactor producing an unstable system, where the non-linearity practically comes into existence.

Based on the obtained values a simple comparison on the performance criterion is shown in the graph below 


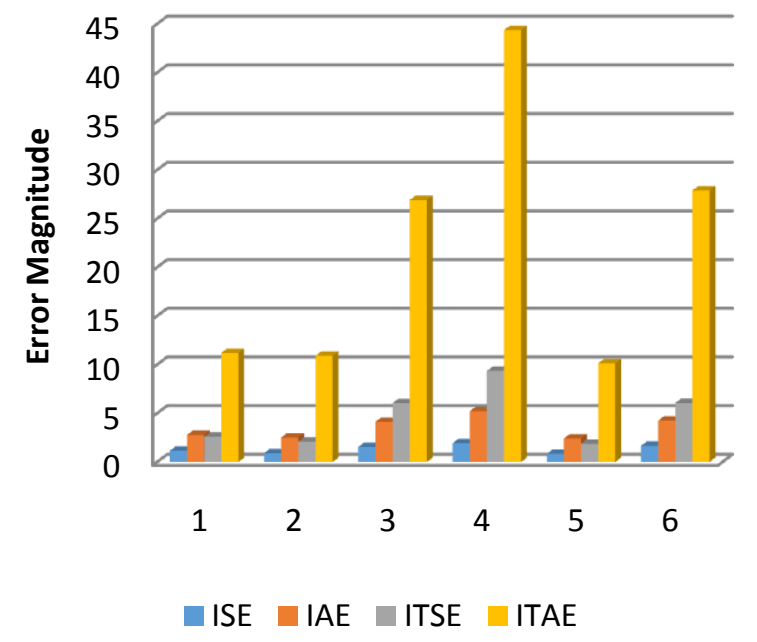

Fig 8: Performance criterion for PSO

The above values shows that the $5^{\text {th }}$ value occurred during a decent iteration produces the error at a less rate when compared with the remaining values. Similarly the performance comparison among the considered algorithms are carried out which is shown in the figure 9.

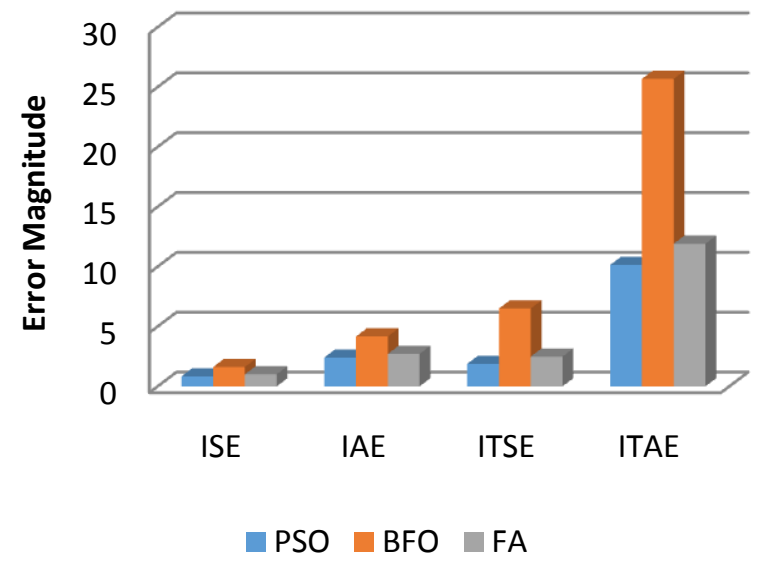

Fig 9: Performance comparison- Growth phase

It can be easily noted from above figure that the PSO algorithm has the least error amongst the three (i.e.) It exhibits good performance criteria when compared to the other two algorithms, namely BFO and Firefly Algorithm for the growth phase of the bioreactor system which exhibits an unstable behavior and this method of tuning tends to be challenging when compared with the stable systems.

\subsubsection{Stability Analysis for the Unstable System:}

Nyquist plots [19] are a great tool in performing stability analysis of closed loop systems. In this work, we have established a closed loop control for the growth phase of the bio-reactor system which is unstable. So, in order to test the stability of this new design, we use the Nyquist plots. They are used in determining the distance from stability of the closed loop systems. The real value of the Nyquist plot lies in the fact that it shows how close the system is to instability. The stable system does not require the Nyquist analysis.

The following Nyquist diagrams depicts the phase margin, gain margin of the closed loop PSO tuned system for an unstable system and it is very clear that the proposed system becomes stable as it has a highly positive phase margin and obeys the Nyquist stability criterion.

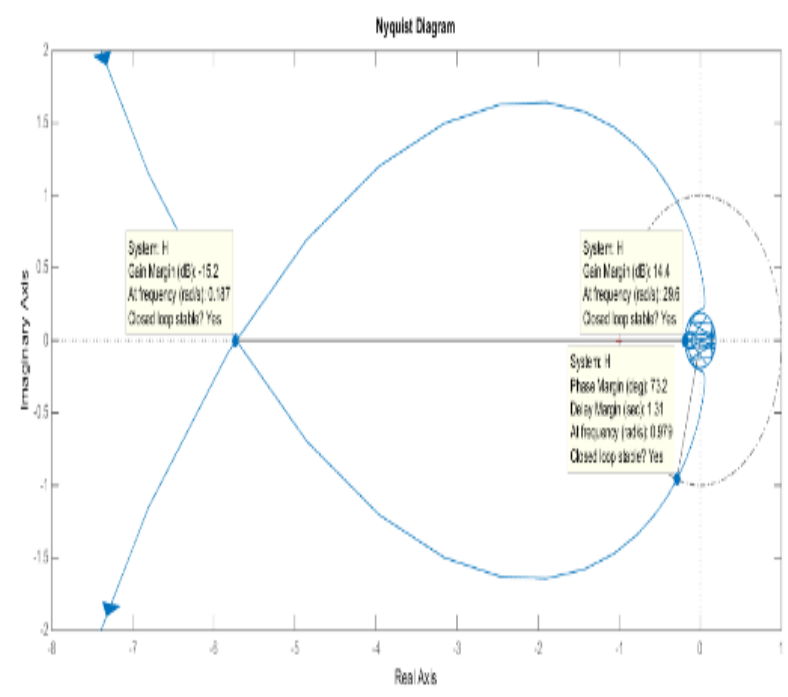

Fig 10: Nyquist plot for PSO tuned system

Since the phase margin and the gain margin plays a vital role in determining the closed loop stability, it is considered as a main parameter for acquiring how close the system is to instability. Similarly the Nyquist plots were drawn for the different algorithms for an unstable system in the growth phase. Due to space constraints we have shown the plot only for the PSO as it provides a greater Phase Margin and Gain Margin comparing with the others algorithms considered here.

Conditions for stability:

$\mathrm{GM}>0($ in $\mathrm{dB}) \Rightarrow$ Closed-loop system is stable.

$\mathrm{GM}>6 \mathrm{~dB}$ is good $\Rightarrow$ we can double the gain without the system becoming unstable.

$\mathrm{PM}>30^{\circ}$ is $\operatorname{good}$ (Note that $\mathrm{PM}=0^{\circ} \rightarrow$ denotes neutrally stable).

\section{CONCLUSION}

Most of the industrial process loops use conventional or modified structure PID controllers. Tuning the controller parameter for time delayed unstable system is a challenging work if the system model is other than a first order plus dead time. In this work, design of optimization based controller tuning for both stable and unstable process models of the bio-reactor has been attempted. The design of controller is formulated as an optimization problem using ISE as the 
performance index. The result obtained from the computer simulation shows that the PSO algorithm provides better result when compared to $\mathrm{BFO}$ and Firefly algorithms for unstable class of systems. Results from stability analysis using Nyquist plots reveal that the tuned systems exhibit good stability by exhibiting a good distance from instability. The proposed method improves the performance of the process in terms of set point tracking, error minimization and better stable operation.

\section{REFERENCES}

[1]. Ahmad Ali and Somanath Majhi., (2006) "Design of Optimum PID Controller by Bacterial Foraging Strategy", Procedings of the IEEE International Conference on Industrial Technology (ICIT), pp.601-605, 2006.

[2]. Arul Ebenezer, E., Abhishek, V., (2013) 'Tuning of PID and modified PID controller by PSO Algorithm for stable and unstable bio-reactor model', IJBRMM- ISSN 22315349- October 2013 issue.

[3]. Chai-ead, N., Aungkulanon, P., and Luangpai- boon, P., Member, IAENG, (2011) "Bees and Firefly Algorithms for Noisy Non-Linear Optimization Problems" Inter- national Multi-conference of Engineers and Computer Scientists, vol 2011.

[4]. Chan-Cheng Chen, Hsia-Ping Huang and Horng-Jang Liaw, (2008) "Set-Point Weighted PID Controller Tuning for Time-Delayed Unstable Processes" Ind.Eng.Chem.Res. Vol.47, No.18, pp. 6983-6990.

[5]. Christian Blum, Maria Jos'eBlesa Aguilera, Andrea Roli, Michael Sampels, (2008) 'An Emerging Approach to Optimization', Springer, vol 2008.

[6]. Farahani, M., Abshouri, A.A., Nasiri, B., and Meybodi, M.R., (2011) 'A Gaussian Firefly Algorithm', International Journal of Machine Learning and Computing, Vol. 1, No. December 2011.

[7]. Hajo Broersma (2011) "Application of the Firefly Algorithm for Solving the Economic Emissions Load Dispatch Problem", Hindawi Publishing Corporation, International Journal of Combinatorics, Volume 2011.

[8]. Kennedy, J., and Eberhart, R., (1995) "Particle swarm optimization, "in Proceedings of the IEEE International Conference on Neural Networks, pp. 1942-1948, December 1995.

[9]. Kumar, S.M.G., Jain, R., Anantharaman, N., Dharmalingam, Y., and Begam, K.M.M.S., (2008) 'Genetic algorithm based PID controller tuning for a model bioreactor', Indian Institute of Chemical Engineers, vol. 50, no. 3, pp. 214-226,

[10]. Manoj, K., Jhunjhunwalaa and Chidambaram, M., (2001) 'PID controller tuning for unstable systems by optimization method', Chemical Engineering Communications Volume 185, Issue 1, pp. 91-113.

[11]. Passino, K.M., (2002) 'Biomimicry of bacterial foraging for distributed optimization and control', IEEE Control Systems Magazine.

[12]. Prashanti, G., and Chidambaram, M., (2000) 'Set-point weighted PID controllers for unstable systems', Journal of the Franklin Institute, Vol 337, Issue 2-3, pp.201-215.

[13]. Rajinikanth, V., and Latha, K., (2010) 'Identification and control of unstable biochemical reactor,' International
Journal of Chemical Engineering Applications, vol. 1, no. 1, pp. 106-111.

[14]. Rajinikanth, V., and Latha, K., (2011) 'Optimization of PID Controller Parameters for Unstable Chemical Systems Using Soft Computing Technique', International Review of Chemical Engineering (I.RE.CH.E.), Vol. 3, N. 3, pp. $350-$ 358.

[15]. Pramod, S., and Chidambaram, M., (2000) 'Closed loop identification of transfer function model for unstable bioreactor for tuning PID controllers', Bioprocess engineering, 22, pp. 185-188.

[16]. Wael M. Korani, Hassen Taher Dorrah, Hassan M. Emara, (2009) 'Bacterial foraging oriented by particle swarm optimization strategy for PID tuning', Proceedings of the 8th IEEE international conference on Computational intelligence in robotics and automation.

[17]. Weng Kee Wong, (2011) 'Nature-Inspired Metaheuristic Algorithms for Generating Optimal Experimental Designs'.

[18]. Yang, X.S., (2010) 'Firefly Algorithm, Stochastic Test Functions and Design Optimisation', Int. J. Bio-Inspired Computation, Vol. 2, No. 2, pp.78-84.

[19]. Brittain, J.E., (2010) 'Electrical Engineering Hall of Fame Harry Nyquist' Vol. 98, No. 8, August 2010 | Proceedings of the IEEE pp. 1535-1537

[20]. Sathe Vivek, M.Chidambaram, "An improved relay auto tuning of PID controllers for unstable FOPTD system", Computers and Chemical Engineering, 29, pp.2060-2068, 2005.

\section{ACKNOWLEDGEMENTS}

We like to show our gratitude to Dr.V.RAJINIKANTH, who showed us the path and future towards the research area.

\section{BIOGRAPHIES}

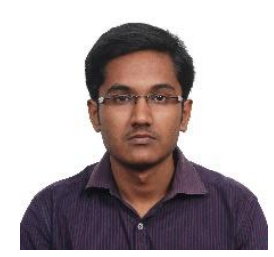

Abhishek V, Under Grad researcher, research enthusiast, St. Joseph's college of engineering, Chennai.

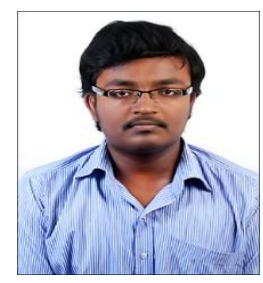

Arul Ebenezer E, Under Grad researcher, St. Joseph's college of engineering, Chennai. 